\title{
Interpolation of the MacAdam ellipses
}

\author{
Emmanuel Chevallier ${ }^{1}$, Ivar Farup ${ }^{2}$ \\ 1: Dept. of Computer science and applied math., Weizmann Institute of Science ; Israel \\ 2: Dept. of computer science, \\ NTNU Norwegian University of Science and Technology; Norway \\ emmanuelchevallier1@gmail.com
}

April 4, 2018

\begin{abstract}
This paper is an attempt to provide a rigorous basis to the interpolation of the MacAdam ellipses. It starts by defining criteria used to compare the different possible interpolations. Then several interpolation strategies are compared. The main conclusion that arises from this comparison is that the ellipses should not be interpolated based on the coefficients of the matrices of the corresponding scalar products, as MacAdam suggested, but on the coefficients of their inverses. It also appears that the $u v$ parameters tend to gives better results than the $x y$ and $a b$ parameters.
\end{abstract}

Keywords: chromaticity space, MacAdam ellipses, Riemannian geometry, interpolation

\section{Introduction}

This paper addresses the study of the geometry of the space of chromaticities, the geometry of interest being the one arising from the human perception. The way the brain compares chromaticities is modeled by a mathematical distance. It is well known that attempts to choose this distance with a Euclidean structure lead to significant distortions of the perception, see [9]. Although there are indications that the space of human colour perception is not even Riemannian [1], it is often assumed to be the case, see [20, 22, 16] among others. There exist various approaches to determine its Riemannian structure. The first consists in deriving the metric from a set of axioms on 
color properties $[18,19]$. Although this yields a consistent mathematical system, assumptions have to be made on the axioms that does not necessarily represent human color vision in limiting cases, such as very low or high intensity light and close to spectral stimuli. Another approach is to construct a more heuristic metric function based on perceptual observations [22]. In this case, it is more difficult to get the perceptual attributes to correspond with the resulting global geometry [20]. Another alternative is to construct a global geometry based on observations from color order experiments [6]. This can give good results for the overall perceptual color attributes, but does not always fit well with local color metric experiments. At last, it is possible model the unit balls of the Riemannian metric by just noticeable difference ellipses, which was suggested by MacAdam and Silberstein, see $[11,21]$. Due to the complexity of the experimental procedure, only 25 ellipses have been measured. As depicts Fig. 1, the ellipses do not cover the whole space.

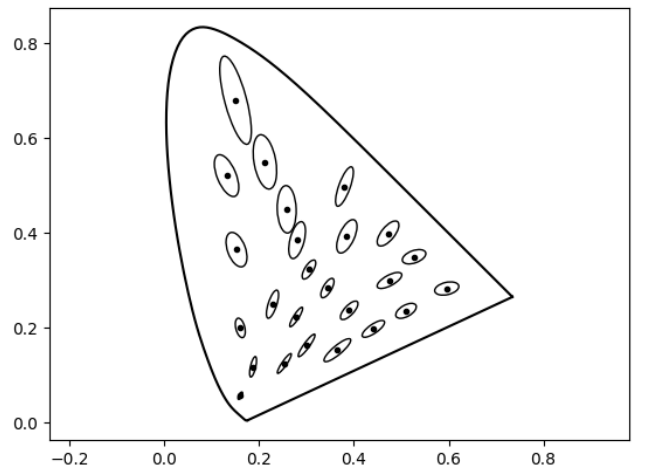

Figure 1: MacAdam ellipses in the $x y$ chromaticity diagram. To ease their visualization, the ellipses are enlarged 10 times.

Thus it is important to be able to interpolate them, see for instance [8]. This paper addresses the problem of the choice of the interpolation strategy. Although these ellipses have been used for many decades, only a very few works paid attention to the interpolation $[12,13,14,5]$. A rigorous study of the choice of the interpolation is more complicated than it seems. When considered as unit balls, the MacAdam ellipses can be expressed in any parametrization of the chromaticity plane. The interpolation can thus be performed in different coordinate systems, which lead to different interpolations. The difficulty is then to compare the quality of the different 
interpolations in a way which depends as little as possible on a coordinate system. The main goal of this paper is to study and propose ways to compare interpolations in a framework as rigorous as possible.

Sections 2 and 3 provide notions on color spaces and Riemannian geometry. Section 4 addresses the problem of the comparison of the interpolations. The comparison tool defined in section 4 is used to compare various interpolation strategies of the MacAdam ellipses in section 5 .

\section{Basics on colors spaces and chromaticities}

Let $s(\lambda)$ be the spectral radiance of a light wave. In the CIE XYZ color space [2], the coordinates $X, Y$ and $Z$ of the color corresponding to $s$ are defined as

$$
\left\{\begin{array}{l}
X=\int s(\lambda) \bar{x}(\lambda) d \lambda \\
Y=\int s(\lambda) \bar{y}(\lambda) d \lambda \\
Z=\int s(\lambda) \bar{z}(\lambda) d \lambda
\end{array}\right.
$$

where $\bar{x}, \bar{y}$ and $\bar{z}$ are real positive functions determined by the $C I E$, see Fig 2.

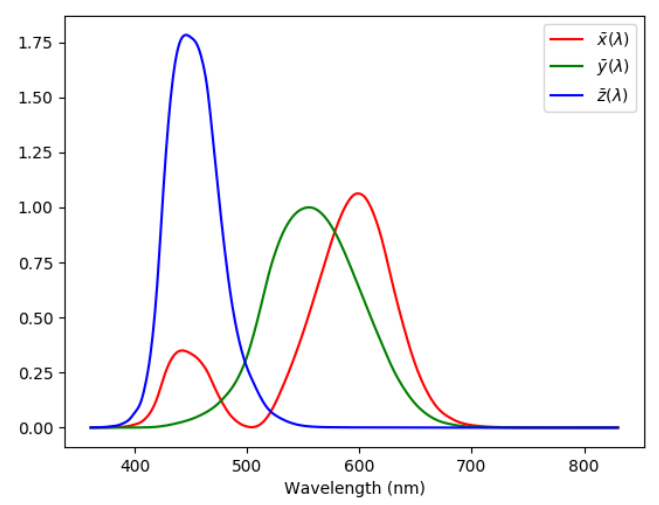

Figure 2: The CIE standard observer color matching functions.

The function $\bar{y}$ models the perception of the luminance: if $s(\lambda)$ is a Dirac at the frequency $\lambda$, the perceived luminance is given by $\bar{y}(\lambda)$.

Consider now the projective plane of the vector space defined by the three coordinates $X, Y$ and $Z$. The chromaticites are defined as the part of the projective plane corresponding to positive distributions $s(\lambda)$. Chromaticities 
are usually parametrized by the $x y$ coordinates defined by the CIE [2] as

$$
\left\{\begin{array}{l}
x=\frac{X}{X+Y+Z} \\
y=\frac{Y}{X+Y+Z}
\end{array} .\right.
$$

A main draw back of the coordinates $X, Y, Z, x$ and $y$ is that they lead to parametrizations whose Euclidean structures do not reflect the visual perception. The Lab and Luv coordinates are non linear transformations of $X, Y$ and $Z$, which have been defined in such a way that their Euclidean structures is closer to the the visual perception,

Lab:

Luv:

$$
\left\{\begin{array}{l}
L=116 f\left(\frac{Y}{Y_{n}}\right)-16 \\
a=500\left(f\left(\frac{X}{X_{n}}\right)-f\left(\frac{Y}{Y_{n}}\right)\right), \\
b=200\left(f\left(\frac{Y}{Y_{n}}\right)-f\left(\frac{Z}{Z_{n}}\right)\right)
\end{array}\right.
$$

$$
\left\{\begin{array}{l}
L=116 f\left(\frac{Y}{Y_{n}}\right)-16 \\
u=13 L\left(\frac{4 X}{X+15 Y+3 Z}-u_{n}^{\prime}\right) \\
v=13 L\left(\frac{9 Y}{X+15 Y+3 Z}-v_{n}^{\prime}\right)
\end{array}\right.
$$

where $\left(X_{n}, Y_{n}, Z_{n}\right)$ are coordinates of a white reference in CIE XYZ, $u_{n}^{\prime}=$ $\frac{4 X_{n}}{X_{n}+15 Y_{n}+3 Z_{n}}, v_{n}^{\prime}=\frac{9 Y_{n}}{X_{n}+15 Y_{n}+3 Z_{n}}$ and

$$
f(t)=\left\{\begin{array}{l}
t^{3} \text { if } t>\left(\frac{6}{29}\right)^{3} \\
\frac{1}{3}\left(\frac{29}{6}\right)^{2} t+\frac{4}{29} \text { otherwise }
\end{array} .\right.
$$

At fixed $L$, it can be checked that there is a homeomorphism between $x y, a b$ and $u v$. Thus $a b$ and $u v$ can be used to parametrize chromaticities. A MacAdam ellipse is an approximation of the set of colors which are indistinguishable to the human eye from a reference color, at constant luminance. These ellipses were introduced by MacAdam in 1942, see [10]. The set of colors of luminance $48 \mathrm{~cd} / \mathrm{m}^{2}$ can be identified with the space of chromaticities and the MacAdam ellipses are then interpreted as sets of chromaticities. When the distance on chromaticities is modeled by a Riemannian distance, it is natural to use the ellipses as unit balls of the local scalar products. Note that since the distances perceived by the brain are not invariant under scaling, there is unfortunately no canonical distance on chromaticities. The metric induced by the MacAdam ellipses is thus associated with the luminance $48 \mathrm{~cd} / \mathrm{m}^{2}$.

The issue tackled in this paper is the interpolation of the ellipses, seen as the local scalar products of a Riemannian distance. In this interpretation the ellipses are naturally represented by 2 by 2 symmetric positive definite matrices. The next two sections provide general considerations on 
the interpolation of the scalar products of a Riemannian distance. These considerations are then put in practice in section 5 , where the underlying space is the space of chromaticities and the local scalar products are the MacAdam ellipses.

\section{Basics of Riemannian geometry}

Let $\mathcal{M}$ be a topological space, homeomorphic to an open subset of $\mathbb{R}^{n}$. An homeomorphism is a continuous map whose inverse is also continuous. Let $\phi$ be an homeomorphism from $\mathcal{M}$ to an open subset $U_{\phi} \subset \mathbb{R}^{n}$. The pair $(\mathcal{M}, \phi)$ is called a chart. A $C^{k}-$ Riemannian metric is a $C^{k}$ field of scalar products on $U_{\phi}$. In other words, a Riemannian metric $(\mathcal{M}, \phi, G)$ associates a matrix $G_{x} \in \operatorname{Sym}^{+}(n)$ to each point $x \in U_{\phi}$, where $\operatorname{Sym}^{+}(n)$ is the set of $n$ by $n$ real symmetric positive definite matrices.

We shall denote $\|u\|_{x}=\sqrt{\langle u, u\rangle_{x}}=\sqrt{G_{x}(u, u)}$. A continuously differentiable path is a map $\gamma:[a, b] \rightarrow \mathcal{M}$ such that $\phi \circ \gamma$ is continuously differentiable. The length of a continuously differentiable path $\gamma$ is defined by the integral

$$
L(\gamma)=\int_{a}^{b}\left\|(\phi \circ \gamma)^{\prime}(t)\right\|_{\phi \circ \gamma(t)} d t .
$$

The infimum of the lengths of the paths joining two points defines a distance between these two points. Geodesics are constant speed paths which are locally shortest paths. Geodesic paths can be seen as straight segments or lines on $\mathcal{M}$.

Let $V$ be an open subset of $\mathbb{R}^{n}$ and $\psi$ an homeomorphism from $\mathcal{M}$ to $V$ such that $\theta=\phi \circ \psi^{-1}$ is a diffeomorphism. Recall that a diffeomorphism is an differentiable homeomorphism whose inverse is also differentiable. Let now $G^{U}$ denotes the field of scalar products defined on $U$. The Riemannian metric $G^{U}$ defined on $U$ can be transported on $V$ by the following transformation,

$$
\forall x \in U, \quad G_{\theta^{-1}(x)}^{V}=d \theta_{x} G_{x}^{U}\left(d \theta_{x}\right)^{T}
$$

where $d \theta_{x}$ is the matrix of the differential of $\theta$ at $x$. It can be checked that the metrics $G^{U}$ and $G^{V}$ define the same distance and area on $\mathcal{M}$.

Let us define a distance between scalar products on $\mathbb{R}^{n}$, independently of a basis. Let $\delta$ be the distance on $\operatorname{Sym}^{+}(n)$ induced by the Riemanian metric $G_{\Sigma}(X, Y)=\operatorname{tr}\left(\Sigma^{-1} X \Sigma^{-1} Y\right)$. Given $A, B \in \operatorname{Sym}^{+}(n)$, the expression of the distance is

$$
\delta(A, B)=\left\|\log \left(A^{-1 / 2} B A^{-1 / 2}\right)\right\|,
$$


where $\log$ is the matrix logarithm and $\|$.$\| is the Frobenius norm on matrices.$ Let $G l_{n}(\mathbb{R})$ denote the $n$ by $n$ real and invertible matrices. $\delta$ is an affineinvariant distance,

$$
\forall P \in G l_{n}(\mathbb{R}), \quad \delta(A, B)=\delta\left(P A P^{T}, P B P^{T}\right) .
$$

Indeed, it is easy to check that $G_{\Sigma}$ is affine-invariant, see [17] for more details. Thus, $\delta$ is invariant by basis change. Let $G_{x}^{U}$ and $\tilde{G}_{x}^{U}$ be two scalar products at $x \in U$. Then,

$$
\delta\left(G_{x}^{U}, \tilde{G}_{x}^{U}\right)=\delta\left(G_{\theta^{-1}(x)}^{V}, \tilde{G}_{\theta^{-1}(x)}^{V}\right) .
$$

However, it is important to note that the comparison between the scalar products at two different points generally depends on the parametrization,

$$
\delta\left(G_{x}^{U}, G_{y}^{U}\right) \neq \delta\left(G_{\theta^{-1}(x)}^{V}, G_{\theta^{-1}(y)}^{V}\right)
$$

since $d \theta_{x}$ and $d \theta_{y}$ are not related. A property of $(\mathcal{M}, \psi)$ is called intrinsic if it invariant under a diffeomorphic change of chart.

\section{Interpolation of a sampled Riemannian metric}

Let $\mathcal{M}$ be a topological space, homeomorphic to an open subset of $\mathbb{R}^{n}$, and let $\phi$ be such a homeomorphism from $\mathcal{M}$ to an open subset $U_{\phi} \subset \mathbb{R}^{n}$. Let $G$ be a Riemannian metric on $(\mathcal{M}, \phi)$. Let $p_{1}, \ldots, p_{k} \in \mathcal{M}^{k}$. Assume that the Riemannian metric is only known at $\left\{\phi\left(p_{1}\right), \ldots, \phi\left(p_{k}\right)\right\}=\left(x_{1}, \ldots, x_{k}\right) \in\left(U_{\phi}\right)^{k}$. We are interested in the question of the interpolation of the Riemannian metric for arbitrary points $p \in \mathcal{M}$. An interpolation rule is a function

$$
\begin{aligned}
R_{\phi}: \quad \cup_{k \in \mathbb{N}}\left(U_{\phi} \times S m^{+}\right)^{k} \times U_{\phi} & \rightarrow \text { Sym }^{+} \\
\left(\left(x_{1}, G_{x_{1}}\right), \ldots,\left(x_{k}, G_{x_{k}}\right), x\right) & \mapsto \hat{G}_{x}
\end{aligned}
$$

To simplify the notations, we drop the subscript $\phi . U_{\phi}$ will be noted $U$ and $R_{\phi}\left(\left(x_{1}, G_{x_{1}}\right), \ldots,\left(x_{k}, G_{x_{k}}\right), x\right)$ will be noted $R(x)$ or $\hat{G}_{x}$.

\subsection{Classical evaluation of an interpolation}

Usually, the quality of an interpolation of a function is judged by its regularity. In our discussion, the notion of regularity includes local and non-local rates of variation. The local regularity notion is represented by the order of differentiability. The non-local notion can be evaluated by quantities such as the Lipschitz constant. Recall that a map $f$ is $\alpha$-Lipschitz if

$$
\forall(x, y), \quad d_{2}(f(x), f(y))<\alpha . d_{1}(x, y)
$$


where $d_{1}$ is a distance on the domain of $f$ and $d_{2}$ a distance on the image. Generally, the evaluation of the non-local regularity requires the existence of distances $d_{1}$ and $d_{2}$. Recall that a Riemannian metric is a function which associates to each $x \in U$ a scalar product $G_{x}^{U}$. In the case of the Riemannian metric interpolation, there is no intrinsic way of evaluating the non-local regularity. Firstly, there is no intrinsic distance on $\mathcal{M}$ other than the unknown metric $G$. In Eq. $2 d_{1}$ is unknown, since it is the interpolated quantity. Secondly, according to the previous discussion there is no intrinsic way of comparing $G_{x}^{U}$ and $G_{y}^{U}$ when $x \neq y$. In Eq. 2, there is no intrinsic $d_{2}$. Among the classic ways of evaluating the interpolation of a function, only the differentiability order can be used in the case of Riemannian metric interpolation. However it is clear that when the metric is known at a finite number of points, there exist many smooth interpolations of the known data. Furthermore, these interpolations can be significantly different. This is formalized in the rest of the paragraph.

Let $G$ be a reference metric on $(\mathcal{M}, \phi)$, such as the perceptual metric in the case of the manifold of colors. Given two Riemannian metrics $G^{1}$ and $G^{2}$, let

$$
D\left(G^{1}, G^{2}\right)=\sup _{x \in U} \delta\left(G_{x}^{1}, G_{x}^{2}\right)
$$

be a distance between $G^{1}$ and $G^{2}$. Note that this distance is intrinsic.

Theorem 4.1. Given a set of points $\left\{x_{1}, \ldots, x_{k}\right\} \in U^{k}$ and a set of scalar products $G_{x_{1}}, \ldots, G_{x_{k}}$, there are infinitly many $\mathcal{C}^{\infty}$ Riemannian metrics $\hat{G}$ such that $\hat{G}_{x_{i}}=G_{x_{i}}$ for all $1 \leq i \leq n$. Furthermore, for all $A \in \mathbb{R}_{+}$, there exist $\mathcal{C}^{\infty}$ metrics $\hat{G}$ and $\hat{G}^{\prime}$ such that $\hat{G}_{x_{i}}=\hat{G}_{x_{i}}^{\prime}=G_{x_{i}}$ and $A<D\left(\hat{G}, \hat{G}^{\prime}\right)$.

Proof. Let $u_{i}=\left\{x_{i}\right\} \cup\left(U \backslash\left\{x_{1}, \ldots, x_{k}\right\}\right)$ and $\left(\alpha_{i}\right)_{i \in\{1, \ldots, k\}}$ be smooth functions such that

$$
\begin{gathered}
\operatorname{supp}\left(\alpha_{i}\right) \subset u_{i}, \\
\forall x \in U, \sum_{i} \alpha_{i}(x)=1 .
\end{gathered}
$$

Let

$$
\hat{G}_{x}=\sum_{i} \alpha_{i}(x) G_{x_{i}} .
$$

Since Sym $^{+}$is convex, $\hat{G}_{x} \in S y m^{+} . \hat{G}$ defines thus a smooth field of $S y m^{+}$ matrices such that $\hat{G}_{x_{i}}=G_{x_{i}}$. By construction, it is possible to impose any arbitrary value $\hat{G}_{y} \in S y m^{+}$at a point $y \notin\left\{x_{i}\right\}$. Hence for all $A>0$, it is possible to construct two smooth metrics $\hat{G}$ and $\hat{G}^{\prime}$ which interpolate the scalar products on the $\left\{x_{i}\right\}$ and such that $D\left(\hat{G}, \hat{G}^{\prime}\right)>A$. 
Two observations can be made. Using the standard evaluations of an interpolation, that is to say the order of differentiability, there are infinitely many interpolations of a Riemannian metric of maximal quality. Furthermore, among these possible interpolations some of them are significantly different. It is thus interesting to try to define additional intrinsic criteria.

\subsection{Adapted intrinsic evaluation}

\subsubsection{Intrinsic criteria}

A possible intrinsic way to evaluate the quality of the interpolation of a set of scalar products is to analyzing the curvature of the interpolated metric. In the considered situation where $\mathcal{M}$ is homeomorphic to $\mathbb{R}^{n}$, interpolations whose curvature have low absolute values are preferred.

Another approach consists in evaluating the interpolation rule itself and not directly the interpolation. Using a "leave one out" cross-validation approach, it is possible to evaluate the generalization capacity of an interpolation rule $R$. Following the discussion of section 3, it is not possible to intrinsically compare the scalar products defined at different points $p \neq q \in \mathcal{M}$. However, it is possible to compare two scalar products defined at the same point independently from a chart. Let $S$ be the set of pairs $\left(x_{i}, G_{x_{i}}\right), 1 \leq i \leq k$. Let $S_{i}$ be the set of pairs obtained by removing the pair $\left(x_{i}, G_{x_{i}}\right)$. It is possible to compare $R\left(S_{i}, x_{i}\right)$ and $G_{x_{i}}$ since they are both defined at the same point. Let $d$ be a distance on $\operatorname{Sym}^{+}(n)$. An interpolation rule $R$ can be evaluated by the set of distances $d\left(R\left(S_{i}, x_{i}\right), G_{x_{i}}\right)$ for all $i$. In the case of the MacAdam ellipses, $d\left(R\left(S_{i}, x_{i}\right), G_{x_{i}}\right)$ is the distance between the ellipse at $p_{i}$ and the interpolated ellipse at $p_{i}$ from the 24 other ellipses. These terms can be seen as playing the role of the evaluation of the non-local regularity of the interpolation. Given a set of pairs $S$, let

$$
Q(R, S)=\sum_{i} d\left(R\left(S_{i}, x_{i}\right), G_{x_{i}}\right)
$$

be a measure of the quality of an interpolation $R$. When $d$ is independent of a chart, this evaluation of the interpolation rule $R$ is intrinsic. It evaluates how well the rule $R$ can predict the measured ellipses.

\subsubsection{Limits of the intrinsic evaluation}

Despite the fact that both previous criteria bring useful information, both suffer from the limitations described in theorem 4.1. This is formalized by theorem 4.2 and 4.3. Although the results are only proved in the two dimensional case, we are convinced that they hold in any dimension. 
Theorem 4.2. Let $U \subset \mathbb{R}^{2}$ and $S$ be a set of $k$ pairs $\left(x_{i}, G_{i}\right) \in U \times \operatorname{Sym}^{+}(2)$. There always exist infinitely many $\mathcal{C}^{\infty}$ interpolations $\hat{G}$ such that $\hat{G}_{x_{i}}=G_{x_{i}}$ for all $1 \leq i \leq k$ and such that $G$ is a flat metric. Furthermore, for all $A \in \mathbb{R}$, there exist $\mathcal{C}^{\infty}$ flat metrics $\hat{G}$ and $\hat{G}^{\prime}$ such that $\hat{G}_{x_{i}}=\hat{G}_{x_{i}}^{\prime}=G_{x_{i}}$ and $A<D\left(\hat{G}, \hat{G}^{\prime}\right)$.

Proof. The idea of the proof is to built a smooth diffeomorphism $f: U \rightarrow V$ whose differential at each $x_{i}$ is $G_{x_{i}}^{1 / 2}$. It can be checked that in the chart $(\mathcal{M}, f \circ \phi), G_{x_{i}}^{V}=I$. The Euclidean metric on $V$ is then a flat metric that interpolates the $G_{x_{i}}^{V}$. The pushforward of this Euclidean metric by $f^{-1}$ provides a flat interpolation of the scalar products $G_{x_{1}}, \ldots, G_{x_{k}}$. The construction of such diffeomorphism is described in the appendix for the dimension 2. Despite the technical difficulties of their constructions, we are convinced that such diffeomorphisms exist in any dimension. Using a similar argument as in theorem 4.1, it is possible to construct interpolations that differ as greatly as possible.

Theorem 4.3 is a simple adaptation of theorem 4.2 .

Theorem 4.3. Let $U \subset \mathbb{R}^{2}$ and $S$ be a set of $k$ pairs $\left(x_{i}, G_{i}\right) \in U \times \operatorname{Sym}^{+}(2)$. There always exist infinitely many interpolation rules $R$ such that $Q(R, S)=$ 0 and such that $\hat{G}$ is $C^{\infty}$ and flat. Furthermore, for all $A \in \mathbb{R}$, there exist two such rules $R$ and $R^{\prime}$ with $A<D\left(\hat{G}, \hat{G}^{\prime}\right)$.

Proof. Let $f$ be a diffeomorphism as in the proof of theorem 4.2. Let $R$ be the rule such that $R(., x)=d f_{x}^{T} d f_{x}$. Let $S$ and $S_{i}$ be as defined in section 4.2.1. Since $R\left(S, x_{i}\right)=R\left(S_{i}, x_{i}\right)=G_{x_{i}}, Q(R, S)=0$. Like in theorem $4.2, R(., x)$ is flat and $C^{\infty}$. Again, using a similar argument as in theorem 4.1, it is possible to construct interpolations that differ as greatly as possible.

As previously, these results tell us two things. Firstly, there are always infinitely many optimal interpolations. Secondly, among these possible interpolations some of them are significantly different. These results are expected to be similar for all the interesting intrinsic criteria.

In practice however, the interpolation should not be evaluated in a fully intrinsic way. In the case of the space of chromaticities under the perceptual Riemannian metric, it is clear that specific parametrizations have to be privileged. Indeed, it is natural to assume that the perceptual metric is related to the physical properties of the eye. On that regard, the interpolations arising in theorem 4.2 and 4.3 are not satisfying since they are based on a chart that have been artificially created and which are unlikely 
to be related to the human perception. The perceptual metric is expected to be related to the CIE XYZ color space. All the color spaces defined by the CIE, or other simple transformations of the CIE XYZ color space are preferred to arbitrary parametrization. The study is restricted to interpolations that have a regular behavior with respect to these charts. Then it is important to dispose of intrinsic criteria to compare them. In the next section, the interpolation are evaluated using the cross validation criteria defined in section 4.2.1.

\section{Interpolation of the MacAdam ellipses}

$\mathcal{M}$ is the space of chromaticities, the points $p_{i} \in \mathcal{M}$ are the chromaticities of the centers of the MacAdam ellipses and the $G_{x_{i}}$ are the scalar products represented by the MacAdam ellipses.

The MacAdam ellipses are assumed to be exact measurements of the local scalar products. Thus all the considered interpolation rules fit exactly the observations: $R\left(S, x_{i}\right)=G_{x_{i}}$. The considered interpolations and their evaluations depend on the followings elements,

- a parametrization of $\mathcal{M}$

- a choice of parameters of the scalar products

- a type of interpolation

- a distance $d$ on the set of scalar products, see Eq. 3 .

\subsection{Color spaces}

Three parametrizations of colors are considered: $x y Y, L a b$ and Luv. At fixed $Y$ there is a unique correspondance between $x y, a b$ and $u v$. In this paper $Y$ is set at $48 \mathrm{~cd} / \mathrm{m}^{2}$ since it is the luminance at which the ellipses are measured. The three couples are reasonable parametrizations of $\mathcal{M}$ in which the different interpolations are computed.

\subsection{Parameters}

Two types of representations of the scalar products are considered.

The first one is based on the matrix of the bilinear form in a chart. Three representations of this matrix are considered, the matrix itself $(M)$, its logarithm $(M L)$ and its inverse $(M I)$. 
The second type of representations is based on the parameters of the ellipses. The parameters of an ellipse are the lengths of its axes $a$ and $b$ with $a \geq b$ and the direction of its main axis. Let $\theta \in[0,2 \pi[$ be an angle between abscissa axis and the main axis as depicted in Fig. 3. A direction is a point on the projective line, which is isometric to a circle. The point representing the direction of the main axis is parametrized by the angle

$$
\alpha=2 .(\theta[\pi]) .
$$

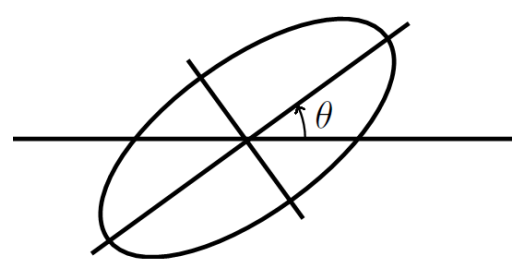

Figure 3: $\theta$ is an angle between the abscissa axis and the main axis of the ellipse. $\alpha=2 .(\theta[\pi])$ parametrizes the direction of the main axis.

Up to a constant, the natural metric of the projective line is the natural metric of a circle parametrized by the angle $\alpha$. As in the matrix case, three representations are considered: $(a, b, \alpha)(P),(\log (a), \log (b), \alpha)(P L)$ and $\left(\frac{1}{a}, \frac{1}{b}, \alpha\right)(P I)$.

\section{$5.3 \quad$ Interpolation type}

Four types of interpolation are considered: nearest neighbor $(N)$, barycentric $(B)$, kernel $(K)$ and spline $(S)$.

In order to define the barycentric and the kernel interpolation, it is necessary to define a notion of average between scalar products. The notion of average is dependent of the choice of parameters used to represent the scalar products. When the parameters have a matrix form $(M-M L-M I)$, the average is chosen to be the standard Euclidean average. When the scalar products are represented by ellipses $(P-P L-P I)$, the average on the first two parameters is also chosen to be Euclidean. The coordinate $\alpha$ represents a point on a circle, the most adapted average is thus the Karcher-Fréchet average ([7]). Since the computation of the Fréchet average requires a gradient descent, we chose instead to compute the Euclidean average and to project the result on the circle. 


\subsubsection{Barycentric}

The barycentric interpolation relies on a triangulation of the centers of the MacAdam ellipses. This triangulation is chosen to be the Delaunay triangulation in the given chart. When the point at which the data is interpolated lies inside a triangle, the interpolated ellipse is a weighted average of the ellipses at the vertices. When the point is outside of the triangulation it is orthogonaly projected on the triangulation. The interpolation is then computed at the projected point. The barycentric interpolation provides a continuous field of ellipses. Note however that as for the nearest neighbor interpolation, this field is not twice differentiable and thus cannot be used to estimate the curvature.

\subsubsection{Kernel}

Given a kernel $k$, the kernel interpolation at $x$ is defined as an average of all the MacAdam ellipses weighted by $\frac{k\left(\left\|x-x_{i}\right\|\right)}{\sum_{j} k\left(\left\|x-x_{j}\right\|\right)}$ where $x_{j}$ are the centers of the MacAdam ellipses and $\|$.$\| is the canonical Euclidean norm of the$ chart. Since the interpolated scalar product at $x_{i}$ should be the observed MacAdam ellipse, the kernel should tend to infinity in zero. The weights are then prolongated by continuity. The function used in the experiments is

$$
k_{\lambda}\left(\left\|x-x_{i}\right\|\right)=\frac{e^{-\frac{\left\|x-x_{i}\right\|}{\lambda}}}{\left\|x-x_{i}\right\|} .
$$

$\lambda$ enables to control the area of influence of each $x_{i}$. In the experiments, $\lambda$ is chosen by cross validation.

\subsubsection{Spline}

The last type of interpolation is based on bi-harmonic splines. For parameters of type $M$ or $P$, the coefficients are interpolated independently. The set of symmetric positive definite matrices is a cone of the symmetric matrices. In practice the bi-harmonic spline interpolation produces matrices outside of this cone, which is incompatible with the scalar product interpretation. Thus only the logarithm of the matrix is used for the spline interpolation. Recall indeed that the logarithm of the cone of symmetric positive definite matrices is the vector space of symmetric matrices. Since it is a circular data, the parameter $\alpha$ encoding the direction of the main axis of the ellipse requires a specific treatment. Instead of using an intrinsic interpolation on the circle, $\alpha$ is represented by a vector $(\cos (\alpha), \sin (\alpha)) \in \mathbb{R}^{2}$ whose coordinates are be interpolated using bi-harmonic splines. The interpolation is then projected on the circle to give the interpolated $\alpha$. 


\subsection{Distance evaluation}

After defining the interpolation rules, we need to define the distance $d$ in the evaluation function $Q$ of Eq. 3,

$$
Q(R, S)=\sum_{i} d\left(R\left(S_{i}, x_{i}\right), G_{x_{i}}\right)
$$

regardless the choice of $d$.

The affine-invariant distance $\delta$ defined in section 3 has the advantage of being independent of the parametrization in which it is computed. However, since the scalar product $G_{x_{i}}$ is the ground truth, it is natural to compare the scalar products in an orthonormal basis of $G_{x_{i}}$. We define two measures of the distance to $G_{x_{i}}$. The first is

$$
d_{x_{i}}^{B}(G)=\int_{G_{x_{i}}(v, v)=1}\left|G(v, v)-G_{x_{i}}(v, v)\right| d v_{x_{i}}
$$

where $d v_{x_{i}}$ is the measure induced by $G_{x_{i}}$ on its unit ball. The second is

$$
d_{x_{i}}^{M}(G)=\left\|M_{G}^{G_{x_{i}}}-I\right\|,
$$

where $M_{G}^{G_{x_{i}}}$ is the matrix of $G$ in an orthonormal basis of $G_{x_{i}}$ and $\|$. the Frobenius norm. We can see that $d_{x_{i}}^{M}$ does not depend on the choice of the orthonormal basis. The upper index $M$ stands for matrix and the upper index $B$ stands for ball. $d_{x_{i}}^{B}$ and $d_{x_{i}}^{M}$ are independent of a chart. The evaluation of the quality of an interpolation rule becomes

$$
Q^{M}(R, S)=\sum_{i} d_{x_{i}}^{M}\left(R\left(S_{i}, x_{i}\right)\right)
$$

and

$$
Q^{B}(R, S)=\sum_{i} d_{x_{i}}^{B}\left(R\left(S_{i}, x_{i}\right)\right)
$$

Note that Eq. 4 and Eq. 5 differ slightly from Eq. 3 since $d$ depends on the ground truth scalar product.

\subsection{Results and discussion}

Tables 1 and 2 summarize the notations defined in the previous section. The quality of the different interpolation rules are gathered in tables 3 and 4 . The two evaluation distances provide similar results. Recall that the spline interpolation in the cone of symmetric positive definite matrices might produce values outside of the cone. Thus splines are used only in the logarithmic representation. 
Independently of the choice of parameters, tables 3 and 4 clearly indicate that interpolations of type $P$ are better than the ones of type $P L$ and $P I$, and that interpolations of type $M I$ are better than the ones of type $M$ and $M L$. These two results are consistent because the lengths of an ellipse axis are the square of the eigenvalues of the inverse of the matrix of the scalar product and not of eigenvalues of the matrix itself. In his 1943 paper, see [11], MacAdam did the interpolation of the coefficients of the matrix of the scalar product in the $x y$ parametrization. It turns out to be the worst setting among the interpolations studied here.

A set of observations can be made about the influence of the parametrization.

- Except the lines $B P-B P L-B P I$ of table 3, $u v$ parameters provide better results than the $a b$ parameters. The plots of errors by ellipses presented in Fig. 5 and 6 and in appendix B show that the ellipse on top of the gamut is always poorly predicted in the $a b$ parametrization. The same ellipse is always properly predicted in the $u v$ parameters.

- Except lines $B P-B P L-S P$ of table 3 and $B M I-B P-B P L-S P$ of table 4 , $u v$ parameters provide better results than the $x y$ parameters.

- Except lines $B M I-B P-B P L-S P$ of table 3 and $4, a b$ parameters provide better results than the $x y$ parameters.

Thus, even if the $u v$ parameters seem to give better results in general, it is not possible to state a clear rule.

$(B P, x y)$ and $(K M I, u v)$ are the two best interpolations according to $Q^{B}$ and $Q^{M}$. It is sensible to privilege $K M I$ in the $u v$ parameters over $B P$ in the $x y$ parameters due to its higher regularity. Unlike the barycentric interpolation, the differentiability of the metric obtained with the kernel interpolation enables to define the Riemannian curvature and the geodesic equations.

Fig. 5 and 6 depicts the individual $d_{x_{i}}^{B}$ scores obtained by each ellipse for interpolations of types $B M-B M I$ and $K M-K M I$. The center of each ellipse is represented by a black circle. For visualization purposes the $d_{x_{i}}^{B}$ scores are interpolated between the ellipses using a barycentric interpolation. Note that again this interpolation is dependent on the parametrization, but it does not deserve a careful study. The colorbar is depicted in Fig. 4.

Fig. 7 depicts the interpolation of the ellipses according to different interpolation strategies: $(K M I, u v),(B P, x y)$ and $(B M, x y) .(K M I, u v)$ and $(B P, x y)$ have the two best scores according to $Q^{B}$ and $Q^{M}$ respectively, while $(B M, x y)$ has the worst scores according to both criteria. It appears 
that the ellipses of the two best score interpolations are reasonably similar. The barycentric frameworks tend to produce poor extrapolations outside of the convex hull of the MacAdam ellipses. Other choices of triangulation of the ellipse centers and their impacts on the quality of the extrapolations should be investigated in future works.

Several geometric aspects of the different interpolations, such as the Riemannian curvature and the geodesics remain to be studied. Given an origin and an initial tangent vector, the kernel and spline interpolations enable establish the geodesic equation. Fig. 8 depicts preliminary results on geodesics. The shape of triangles is characteristic of negatively curved space, which confirms the results obtained in [4]. Recall that since the curvature of the space is not expected to be uniformly negative, the geodesics are not expected to be unique. Even though the geodesics joining two fixed points tend to follow similar trends, a more in depth analysis of the different region of the gamut should be performed in a future work. 
Table 1: Interpolation type

\begin{tabular}{|c|c|}
\hline N & nearest neighbor \\
\hline B & barycentric \\
\hline K & kernel \\
\hline S & spline \\
\hline
\end{tabular}

Table 2: Interpolation parameters

\begin{tabular}{|c|c|}
\hline $\mathrm{M}$ & matrix \\
\hline $\mathrm{P}$ & ellipse parameters \\
\hline $\mathrm{L}$ & logarithm \\
\hline $\mathrm{I}$ & inverse \\
\hline
\end{tabular}

Table 3: Evaluation of the different Table 4: Evaluation of the different interpolation rules using $Q^{B}$.

\begin{tabular}{|c|c|c|c|}
\hline & $x y Y$ & Lab & Luv \\
\hline$N$ & 0.292 & 0.256 & 0.216 \\
\hline$B M$ & 0.337 & 0.228 & 0.227 \\
\hline$B M L$ & 0.178 & 0.178 & 0.171 \\
\hline$B M I$ & 0.168 & 0.185 & 0.165 \\
\hline$B P$ & 0.16 & 0.186 & 0.198 \\
\hline$B P L$ & 0.183 & 0.193 & 0.198 \\
\hline$B P I$ & 0.225 & 0.205 & 0.208 \\
\hline$K M$ & 0.293 & 0.242 & 0.199 \\
\hline$K M L$ & 0.247 & 0.207 & 0.174 \\
\hline$K M I$ & 0.205 & 0.195 & 0.163 \\
\hline$K P$ & 0.229 & 0.204 & 0.18 \\
\hline$K P L$ & 0.246 & 0.211 & 0.185 \\
\hline$K P I$ & 0.265 & 0.219 & 0.192 \\
\hline$S M L$ & 0.265 & 0.208 & 0.178 \\
\hline$S P$ & 0.175 & 0.207 & 0.184 \\
\hline$S P L$ & 0.254 & 0.215 & 0.184 \\
\hline$S P I$ & 0.263 & 0.2 & 0.188 \\
\hline
\end{tabular}
interpolation rules using $Q^{M}$.

\begin{tabular}{|c|c|c|c|}
\hline & $x y Y$ & Lab & Luv \\
\hline$N$ & 1.461 & 1.232 & 0.918 \\
\hline$B M$ & 1.814 & 1.024 & 0.971 \\
\hline$B M L$ & 0.792 & 0.769 & 0.7 \\
\hline$B M I$ & 0.624 & 0.738 & 0.644 \\
\hline$B P$ & 0.62 & 0.799 & 0.79 \\
\hline$B P L$ & 0.74 & 0.854 & 0.823 \\
\hline$B P I$ & 0.956 & 0.931 & 0.87 \\
\hline$K M$ & 1.514 & 1.121 & 0.809 \\
\hline$K M L$ & 1.128 & 0.941 & 0.705 \\
\hline$K M I$ & 0.776 & 0.795 & 0.602 \\
\hline$K P$ & 1.004 & 0.911 & 0.721 \\
\hline$K P L$ & 1.118 & 0.963 & 0.761 \\
\hline$K P I$ & 1.242 & 1.006 & 0.799 \\
\hline$S M L$ & 1.440 & 0.894 & 0.706 \\
\hline$S P$ & 0.665 & 0.863 & 0.694 \\
\hline$S P L$ & 1.232 & 0.931 & 0.715 \\
\hline$S P I$ & 1.308 & 0.826 & 0.7 \\
\hline
\end{tabular}




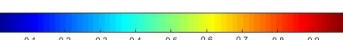

Figure 4: Colorbar

(xy)
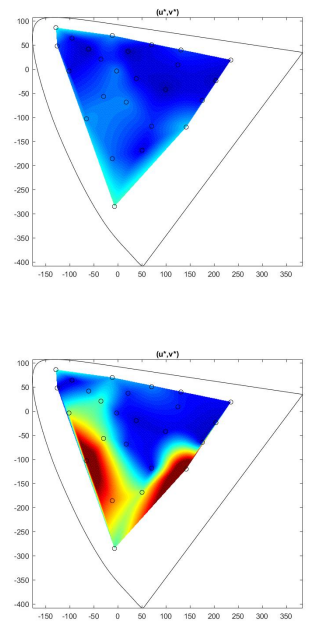

$(\mathrm{ab})$
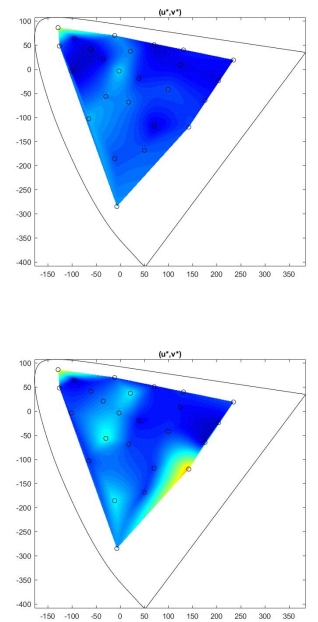

(uv)
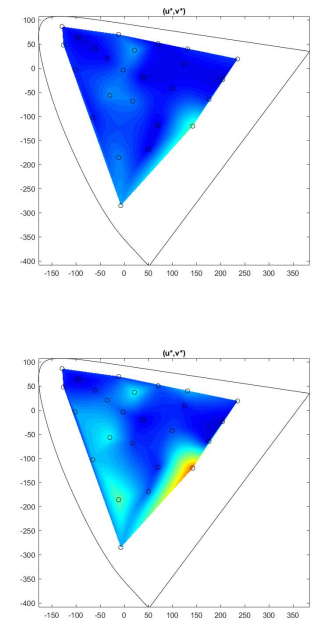

Figure 5: Error maps where the error between the interpolated ellipses and the true ellipses are measured by $d_{x_{i}}^{B}\left(\hat{G}_{x_{i}}\right)$. The error is interpolated between the $x_{i}$ using the barycentric interpolation. First row: BMI, second row: BM.

(xy)
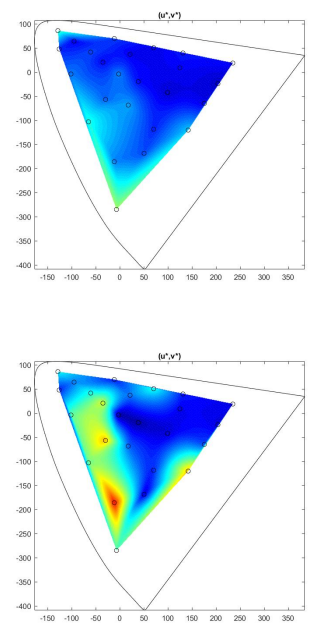

$(\mathrm{ab})$
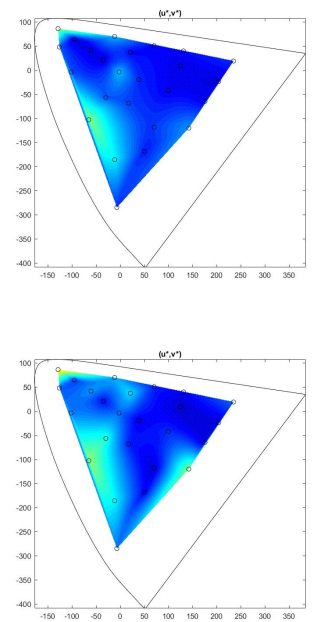

(uv)
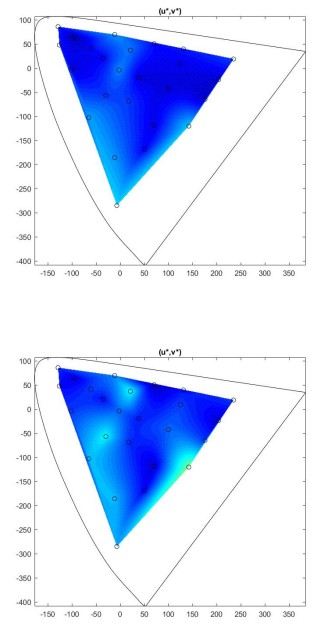

Figure 6: Same errors as in Fig. 5 for different interpolations. First row: KMI, second row: KM 


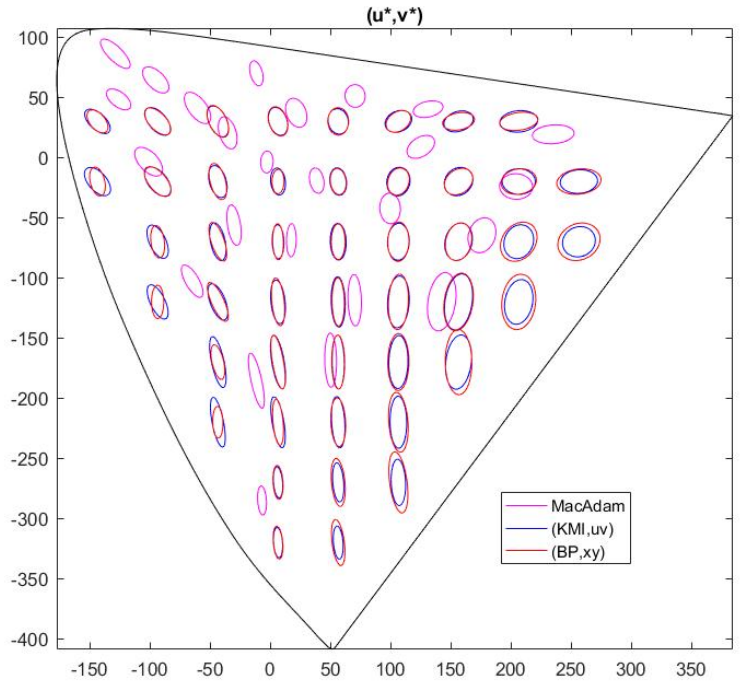

(a)

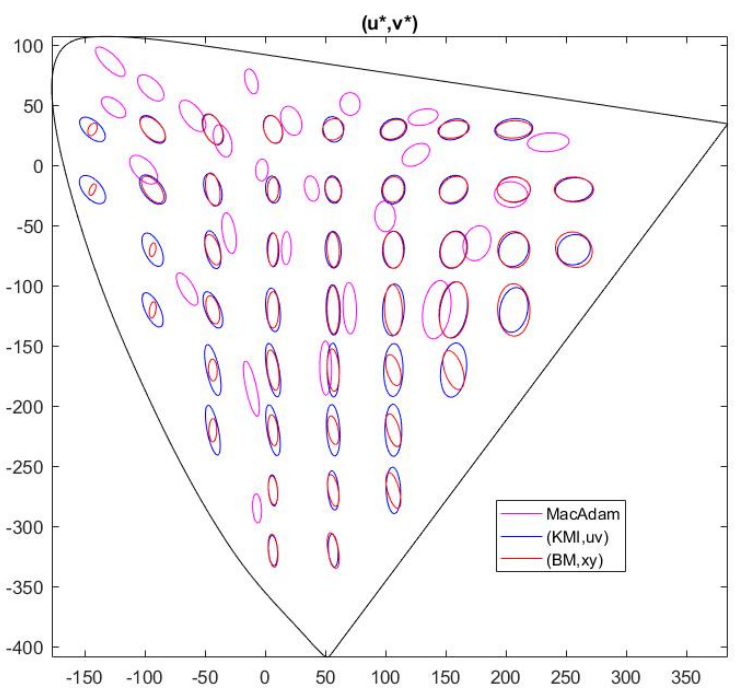

(b)

Figure 7: Plots of ellipses in $u v$ coordinates, (a): best interpolations for $d^{B}$ and $d^{M},(\mathrm{~b})$ : a best interpolation for $d_{x_{i}}^{B}$ together with the worst interpolation for both distances. 


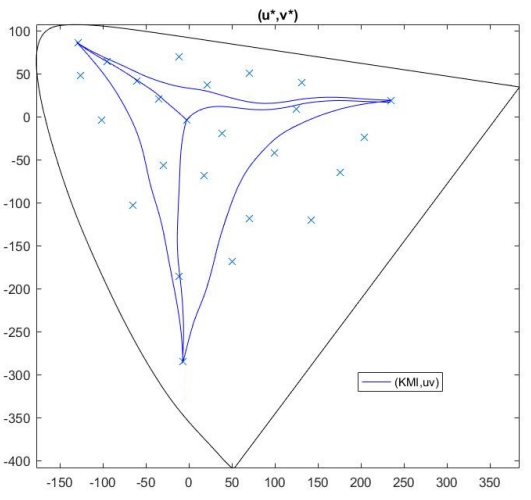

(a)

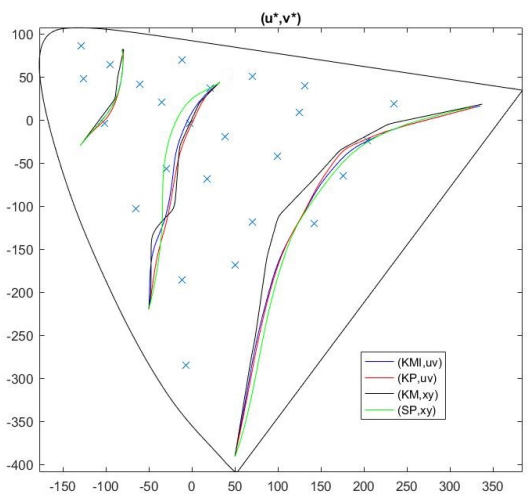

(c)

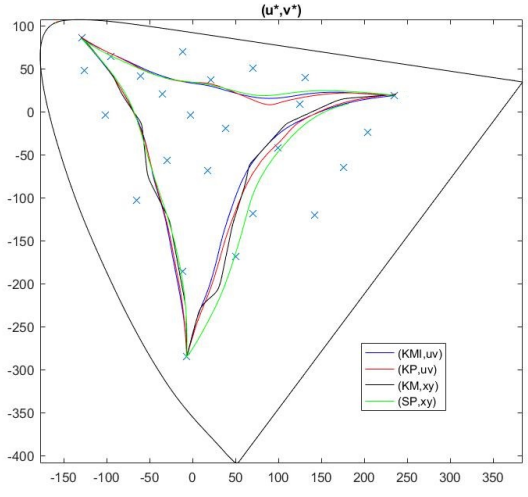

(b)

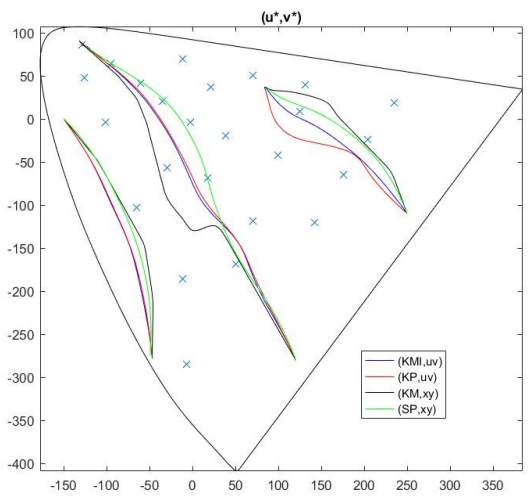

(b)

Figure 8: Geodesics joining fixed points are computed using the geodesic equation and by adjusting the initial tangent vector. Although only one geodesic per pairs of points and interpolations is plotted, recall that the uniqueness of the geodesics is not guaranteed. (a): geodesics joining centers of the central and the extremal MacAdam ellipses using the (KMI,uv) interpolation. (b): geodesics joining centers of extremal ellipses according to different interpolations. The $(K M, x y)$ upper geodesics is missing due to some numerical instabilities. (b,c,d): although the $(K M I, u v)$ and $(K P, u v)$ geodesics are usually very close one from another, it is not the case in the upper right corner of the $u v$ gamut. 


\section{Conclusion}

There exist several sensible parametrizations of the chromaticity plane. In this paper we focused on parameters $x y$ and their transformation in the $a b$ and $u v$ planes at $48 \mathrm{~cd} / \mathrm{m}^{2}$. A set of simple interpolation strategies of the MacAdam ellipses described in section 5 are considered in each parametrization. In order to determine in which of these coordinates the interpolation should be achieved, it is necessary to dispose of an intrinsic criteria, that is to say a criteria independent of the choice of a parametrization. Curvature and cross-validation are examples of such criteria. Theorems 4.2 and 4.3 show that when the set of scalar products is known on a finite set, there are always infinitely many interpolations which are optimal for both criteria.

This result indicates that a purely intrinsic evaluation would lead to irrelevant results and that it is thus important to study only interpolations which are regular with respect to the natural parametrizations.

Cross-validation is used to compare the different interpolations described in section 5, the results are summarized in tables 3 and 4 . The main information that can be pulled out of these tables is that the interpolation of the ellipses should be made on the parameters of the ellipses or on the inverse of the matrix of the corresponding scalar product and not directly on the matrix of the scalar product as MacAdam suggested in [11]. Although this is not the case for all interpolations, the $u v$ parameters tend to present better results.

The experiments presented in this paper are based on pointwise comparisons of interpolated ellipses. On a local level, the two best interpolations provide consistent results: the differences between the ellipses are small. Our future work will mainly focus on the study of the curvature and on the geodesics of the interpolated metrics. Since all the constructions can be generalized to three dimensions, we also intend to analyze of the ellipsoids datasets [15] describing the geometry of the full color space. At last we plan to carry on our work on statistics on the manifold of colors, see [3].

\section{A Construction of the diffeomorphism of theorems 4.2 and 4.3}

Given $x_{1}, . ., x_{k}$ in $\mathbb{R}^{n}$ and $A_{1}, . ., A_{k}$ a set of $n \times n$ symmetric positive definite matrices, we search for a smooth diffeomorphism $f$ of $\mathbb{R}^{n}$ such that $d f_{x_{i}}=A_{i}$. Let $R$ be such that the closed balls $B\left(x_{i}, R\right)$ do not intersect. We construct a diffeomorphism $f$ which is equal to the identity outside of the $B\left(x_{i}, R\right)$. 
The main step is to find a diffeomorphism $g$ which is the identity outside of the ball $B(0, R)$ such that $d g=A$ for an arbitrary symmetric positive definite matrix $A$. Then, using the translations $t_{i}: x \rightarrow x+x_{i}$, we obtain $k$ diffeomorphisms $g_{i}=t_{i} \circ g_{A_{i}} \circ t_{i}^{-1}$ that we glue together to obtain the diffeomorphism $f=g_{1} \circ \ldots \circ g_{k}$. Since the balls $B\left(x_{i}, R\right)$ are disjoint, we have that $f$ is equal to $g_{i}$ in each ball $B\left(x_{i}, R\right)$, hence $d f_{x_{i}}=A_{i}$.

Construction of $g_{A}$. By a linear change of coordinate we can suppose that the matrix $A$ is diagonal with positive coefficients. We start by the one dimensional case.

Let $a$ be a positive number. Set $\phi: \mathbb{R} \rightarrow \mathbb{R}$ be a $C^{\infty}$ even function such that $\phi(0)=1 \geq \phi(x) \geq-\epsilon$ for all $x$ in $\mathbb{R}, \int_{0}^{R} \phi(t) d t=0, \int_{0}^{R}|\phi(t)| d t \leq R / 10$ and $\sup \phi \subset[-R, R]$. The constant $\epsilon$ will be chosen later. Set

$$
g_{a}(x)=\varphi(a, x)=\int_{0}^{x}(1+(a-1) \phi(t)) d t .
$$

- i. $g$ is $C^{\infty}$ and $g_{a}^{\prime}(x)=1+(a-1) \phi(x)$, hence $g_{a}^{\prime}(0)=a$.

- ii. Since $\int_{0}^{R} \phi(t) d t=0$, we have $g_{a}(x)=x+(a-1) \int_{0}^{x} \phi(t) d t=x$ when $|x| \geq R$.

- iii. Let us now choose the constant $\epsilon$ such that $g_{a}^{\prime}(x)>0$ for all $x$. When $a \geq 1$, we have $1+(a-1) \phi(x) \geq \frac{1}{2}$ iff $\phi(x) \geq \frac{-1}{2(a-1)}$, it is then enough to take $\epsilon=\frac{1}{2(a-1)}(\epsilon=\infty$ if $a=1)$. When $a \leq 1$, $1+(a-1) \phi(x) \geq a$ iff $(1-a)(1-\phi(x)) \geq 0$ which is true because $\phi \leq 1$.

Therefore $g_{a}$ is a diffeomorphism such that $g_{a}^{\prime}(0)=a$ and $g_{a}(x)=x$ when $|x| \geq R$.

In dimension 2, the idea is to take $F\left(x_{1}, x_{2}\right)=\left(\varphi\left(a_{1}, x_{1}\right), \varphi\left(a_{2}, x_{2}\right)\right)$. This almost works but $F$ is not the identity outside of $[-R, R] \times[-R, R]$. We have $F\left(x_{1}, x_{2}\right)=\left(x_{1}, x_{2}\right)$ when $\left|x_{1}\right|$ and $\left|x_{2}\right| \geq R$ while we would like the same result when $\left|x_{1}\right|$ or $\left|x_{2}\right| \geq R$. We modify the function $F$ by varying $a_{1}$ and $a_{2}$. We consider even $\mathcal{C}^{\infty}$ functions $\alpha_{i}, i=1,2$, such that

$\alpha_{i}(x)=\left\{\begin{array}{l}a_{i} \text { for } x \in[0, R] \\ \text { monotonous on }[R, 2 R] \text { with derivative } \leq 2 a_{i} / r \text { in absolute value } . \\ 1 \text { for } x \geq 2 R\end{array}\right.$.

The map we are looking for is defined by

$$
G\left(x_{1}, x_{2}\right)=\left(\varphi\left(\alpha_{1}\left(x_{2}\right), x_{1}\right), \varphi\left(\alpha_{2}\left(x_{1}\right), x_{2}\right)\right)
$$

Since $G$ coincides with $F$ on $[-R, R] \times[-R, R]$ the differential in 0 is the diagonale $\left(a_{1}, a_{2}\right)$. By construction $G$ is the identity outside of the square 
$[-2 R, 2 R] \times[-2 R, 2 R]$. It remains to check that $G$ is a diffeomorphism. It is enough to prove that $G$ is injective and that its Jacobian is always positive. Indeed, the surjectivity is implied by the injectevity and the fact that $G$ is the identity outside of the square $[-2 R, 2 R] \times[-2 R, 2 R]$. Let us start with the injectivity. Assume that $G\left(x_{1}, x_{2}\right)=G\left(y_{1}, y_{2}\right)$.

Remark: $\left|x_{1}\right| \geq R$ is equivalent to $\left|y_{1}\right| \geq R$. Indeed $\left|x_{1}\right| \geq R$ and $\left|y_{1}\right|<R$ is not possible because we would have $\left|\varphi\left(\alpha_{1}\left(x_{2}\right), x_{1}\right)\right| \geq R$ and $\left|\varphi\left(\alpha_{1}\left(y_{2}\right), y_{1}\right)\right|<R$.

Case $1\left|x_{1}\right| \geq R$. Then we also have $\left|y_{1}\right| \geq r$ and thus $G\left(x_{1}, x_{2}\right)=$ $\left(x_{1}, \varphi\left(\alpha_{2}\left(x_{1}\right), x_{2}\right)\right)=G\left(y_{1}, y_{2}\right)=\left(y_{1}, \ldots\right)$ thus $y_{1}=x_{1}$. We deduce that $\varphi\left(\alpha_{2}\left(y_{1}\right), y_{2}\right)=\varphi\left(\alpha_{2}\left(x_{1}\right), y_{2}\right)=\varphi\left(\alpha_{2}\left(x_{1}\right), x_{2}\right)$ and thus that $y_{2}=y_{1}$ because the functions $x \rightarrow \varphi(a, x)$ are injective.

Case $2\left|x_{1}\right|<R$. According to the remark $\left|y_{1}\right|<R$. We need to discuss now the second variable.

Sub-case 1. $\left|x_{2}\right| \geq R$ comes down to case 1 by changing variables 1 and 2 .

Sub-case 2. $\left|x_{2}\right|<R$. Using the remark we also have $\left|y_{2}\right|<R$. Therefore $G\left(x_{1}, x_{2}\right)=\left(\varphi\left(a_{1}, x_{1}\right), \varphi\left(a_{2}, x_{2}\right)\right)=\left(\varphi\left(a_{1}, y_{1}\right), \varphi\left(a_{2}, y_{2}\right)\right)$ and thus $\left(x_{1}, x_{2}\right)=\left(y_{1}, y_{2}\right)$.

\section{Jacobian}

$$
\partial_{x_{1}} G\left(x_{1}, x_{2}\right)=\left(1+\left(\alpha_{1}\left(x_{2}\right)-1\right) \psi\left(x_{1}\right), \alpha_{2}^{\prime}\left(x_{1}\right) \int_{0}^{x_{2}} \psi(t) d t\right)
$$

and

$$
\partial_{x_{2}} G\left(x_{1}, x_{2}\right)=\left(\alpha_{1}^{\prime}\left(x_{2}\right) \int_{0}^{x_{1}} \psi(t) d t, 1+\left(\alpha_{2}\left(x_{1}\right)-1\right) \psi\left(x_{2}\right)\right)
$$

thus

$$
\begin{array}{r}
\text { Jac } G\left(x_{1}, x_{2}\right)=\left(1+\left(\alpha_{1}\left(x_{2}\right)-1\right) \psi\left(x_{1}\right)\right)\left(1+\left(\alpha_{2}\left(x_{1}\right)-1\right) \psi\left(x_{2}\right)\right) \\
-\alpha_{1}^{\prime}\left(x_{2}\right) \int_{0}^{x_{1}} \psi(t) d t \alpha_{2}^{\prime}\left(x_{1}\right) \int_{0}^{x_{2}} \psi(t) d t .
\end{array}
$$

Using inequalities of item iii, and then the inequality $\int_{0}^{R}|\phi(t)| d t \leq R / 10$, we obtain

$$
\begin{aligned}
\operatorname{Jac} G\left(x_{1}, x_{2}\right) & \geq \min \left\{a_{1}, \frac{1}{2}\right\} \min \left\{a_{2}, \frac{1}{2}\right\}-\left|\alpha_{1}^{\prime}\left(x_{2}\right) \alpha_{2}^{\prime}\left(x_{1}\right)\right|\left(\int_{0}^{R}|\psi(t)| d t\right)^{2} \\
& \geq \min \left\{a_{1}, \frac{1}{2}\right\} \min \left\{a_{2}, \frac{1}{2}\right\}-\left(4 a_{1} a_{2} / R^{2}\right) R^{2} / 100 \\
& >0 .
\end{aligned}
$$




\section{B Error maps}

These plots depict the $d^{B}$ error per ellipses for several interpolations. The error is interpolated between the ellipses as described in paragraph 5.5. Notations are defined in tables 1 and 2. Fig. 9 provides the color code.

Figure 9: Colorbar

\section{B.1 $\mathrm{MI} / \mathrm{M}-\mathrm{P} / \mathrm{PI}$}

\section{B.1.1 MI/M}

(xy)
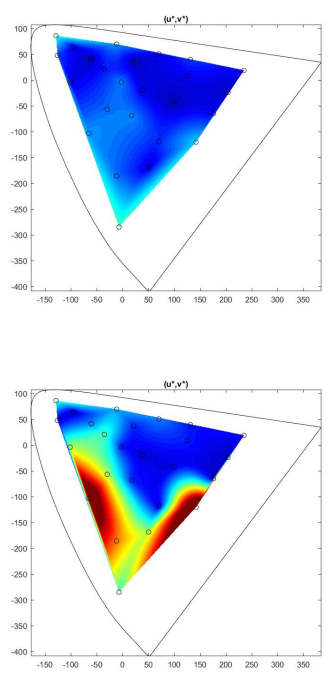

(ab)
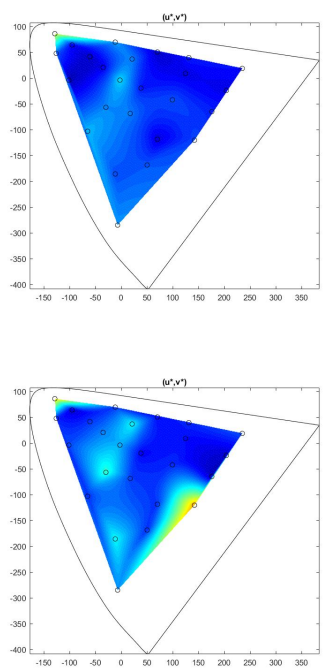

(uv)
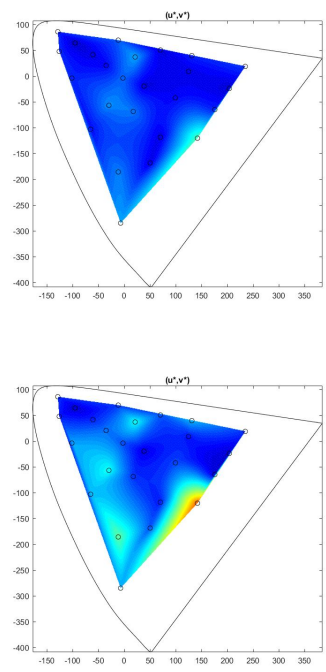

Figure 10: Barycentric 
(xy)
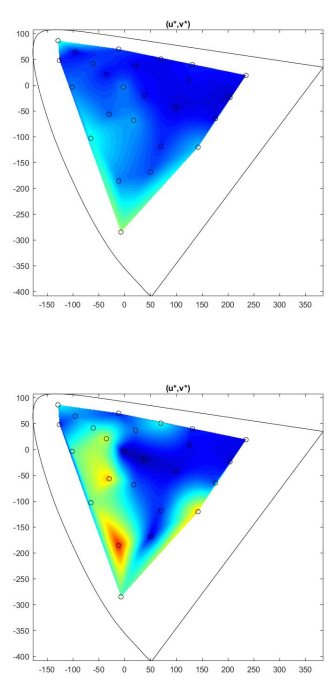

(ab)
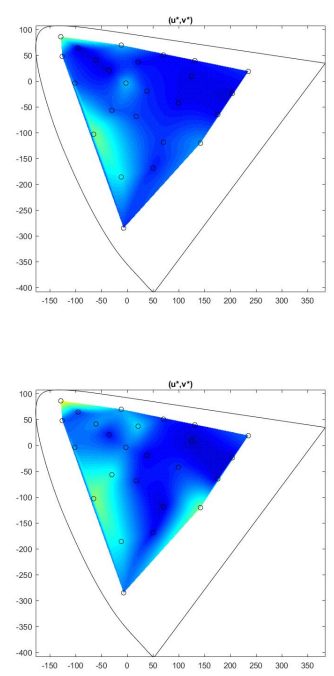

Figure 11: Kernel

\section{B.1.2 P/PI}

(xy)
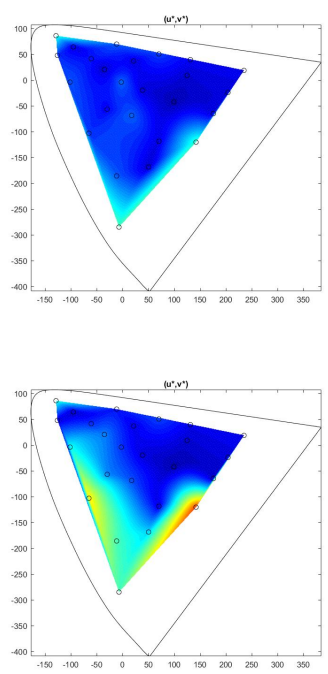

(ab)
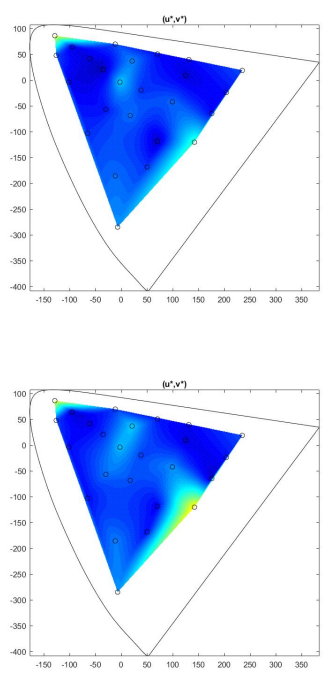

(uv)
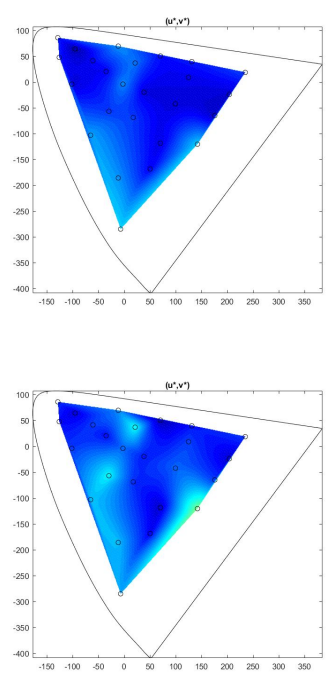

(uv)
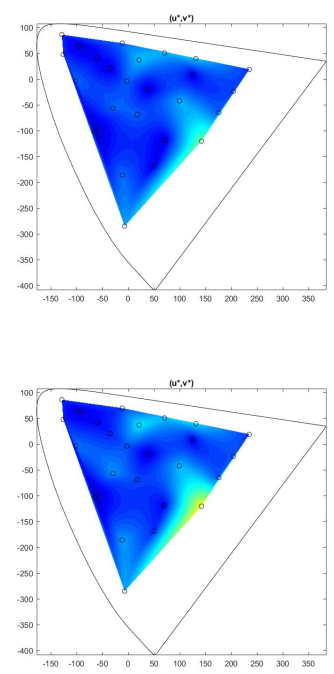

Figure 12: Barycentric 
(xy)
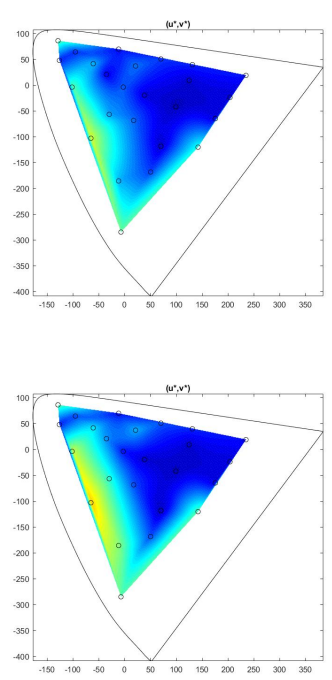

(ab)
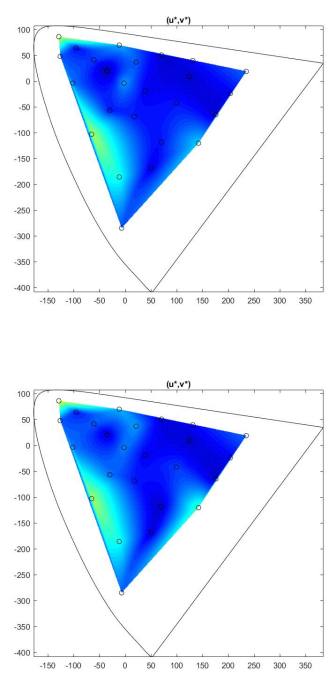

Figure 13: Kernel

\section{B.2 MI/P}

(xy)
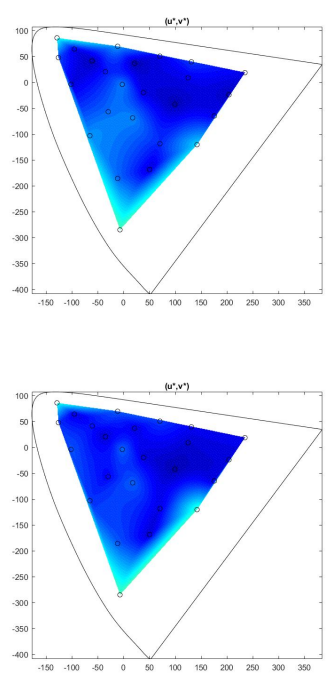

(ab)
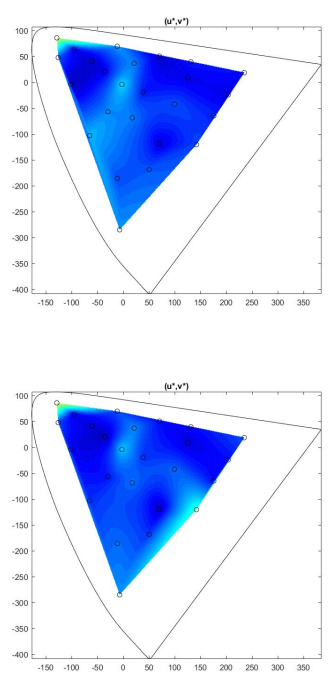

(uv)
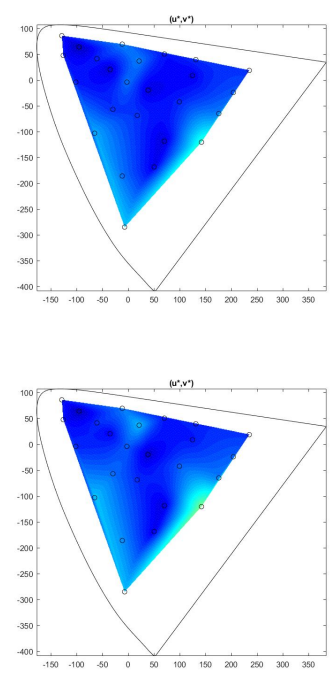

Figure 14: Barycentric 
(xy)
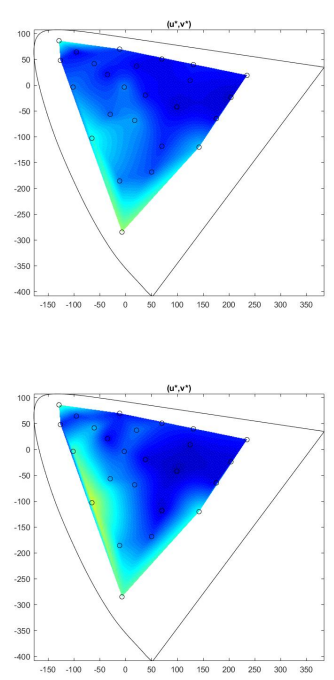

(ab)
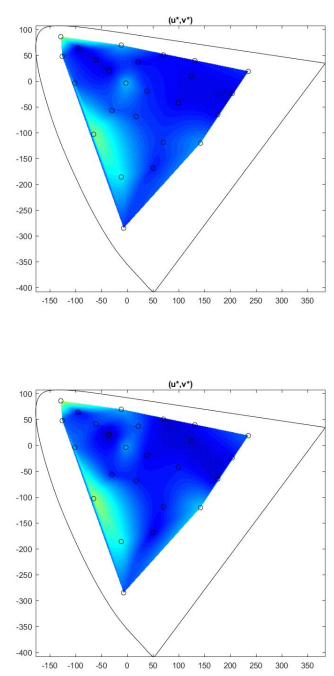

Figure 15: Kernel (uv)
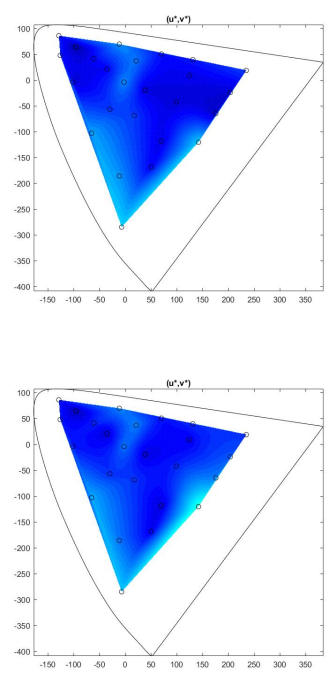

\section{B.3 B $/ \mathrm{K} / \mathrm{S}$}

(xy)
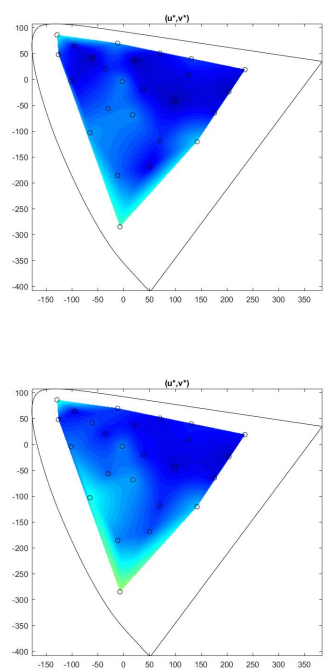

(ab)
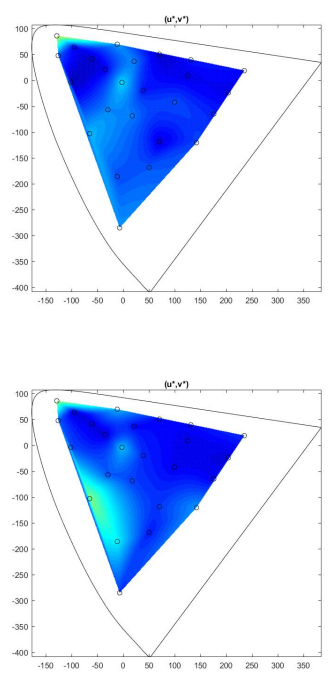

(uv)
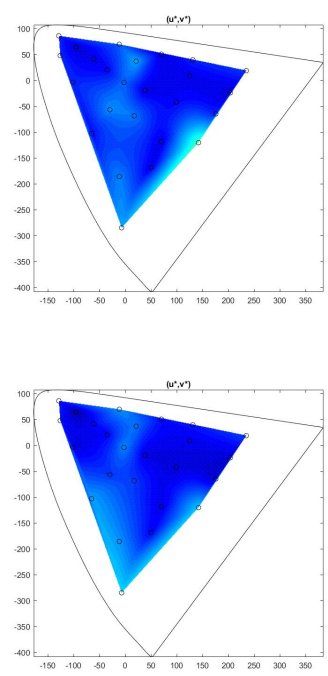

Figure 16: MI (without splines) 
(xy)
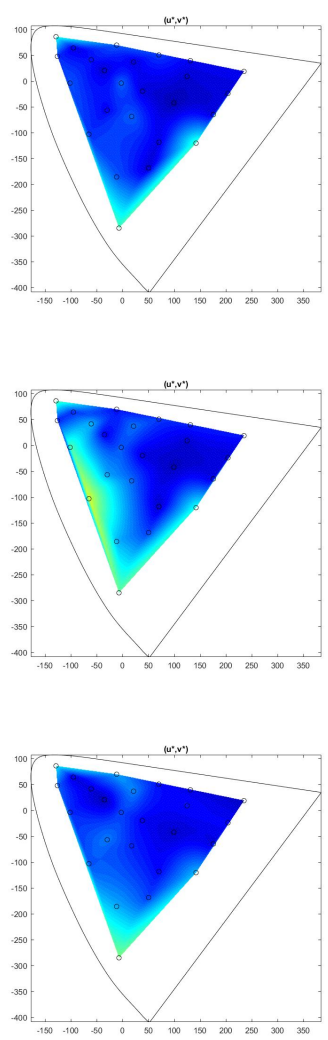

(ab)
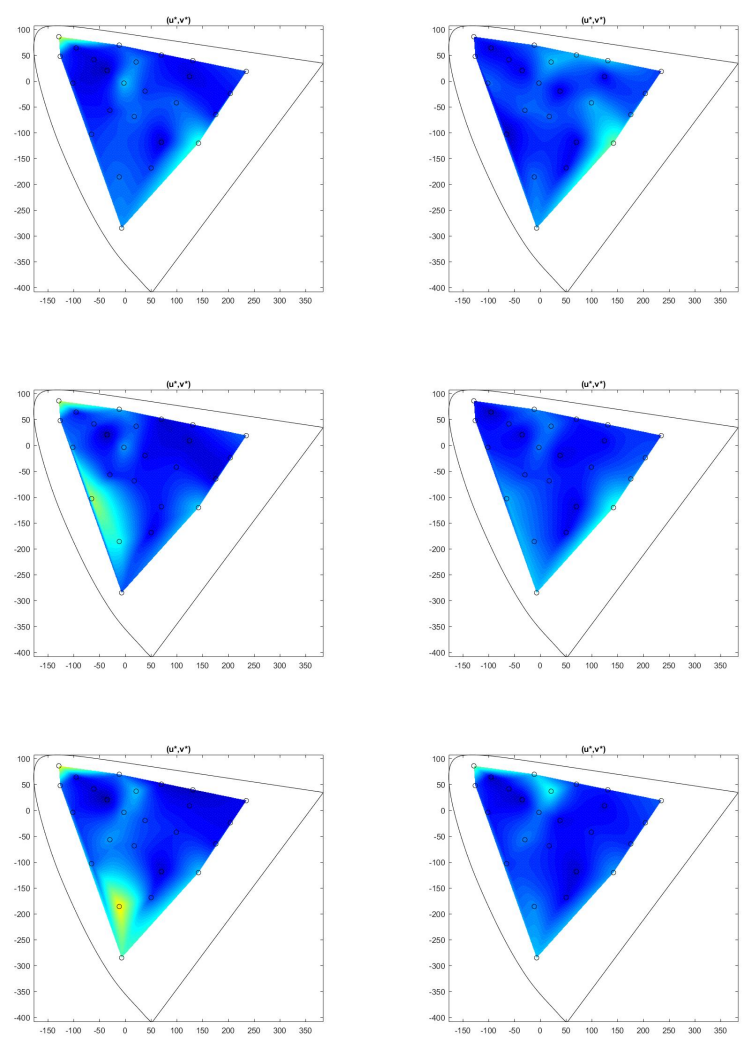

(uv)
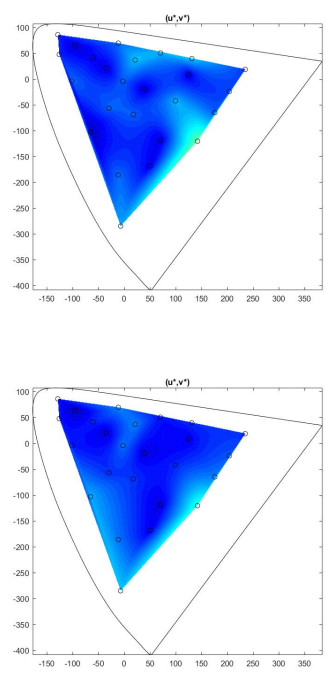

Figure 17: P

\section{References}

[1] Alleysson, D., and Méary,D.. Neurogeometry of color vision. Journal of Physiology-Paris 106.5 (2012): 284-296.

[2] ISO 11664-1/CIE S 014-1/E:2007: CIE Standard Colorimetric Observers

[3] Chevallier, E., Farup, I., Angulo, J. (2015, October). Histograms of images valued in the manifold of colours endowed with perceptual metrics. In International Conference on Networked Geometric Science of Information (pp. 762-769). Springer, Cham.

[4] Farup, I. (2014). Hyperbolic geometry for colour metrics. Optics Express, 22(10), 12369-12378. 
[5] Gravesen, J. (2015). The metric of colour space. Graphical Models, $82,77-86$.

[6] Judd, Deane B. Ideal color space: Curvature of color space and its implications for industrial color tolerances. Palette 29.21-28 (1968): $4-25$.

[7] Karcher, H. (1977). Riemannian center of mass and mollifier smoothing. Communications on pure and applied mathematics, 30(5), 509541.

[8] Kohei, T., Chao, J., and Lenz, R. (2010, January). On curvature of color spaces and its implications. In Conference on Colour in Graphics, Imaging, and Vision (Vol. 2010, No. 1, pp. 393-398). Society for Imaging Science and Technology.

[9] Kuehni, Rolf G., and Andreas Schwarz. Color ordered: a survey of color systems from antiquity to the present. Oxford University Press, 2008.

[10] MacAdam, D. L. (1942). Visual sensitivities to color differences in daylight. JOSA, 32(5), 247-274.

[11] MacAdam, D. L. (1943). Specification of small chromaticity differences. Josa, 33(1), 18-26.

[12] MacAdam, D. L. (1957). Analytical approximations for color metric coefficients. JOSA, 47(4), 268-274.

[13] MacAdam, D. L. (1964). Analytical approximations for color metric coefficients. II. Friele approximation. JOSA, 54(2), 249-256.

[14] MacAdam, D. L. (1964). Analytical Approximations for Color Metric Coefficients. III. Optimization of Parameters in Frieles Formulas. JOSA, 54(9), 1161-1165.

[15] Melgosa, M., et al. "Suprathreshold color-difference ellipsoids for surface colors." Color Research and Application 22.3 (1997): 148-155.

[16] Pant, Dibakar Raj, and Ivar Farup. "Riemannian formulation and comparison of color difference formulas." Color Research and Application 37.6 (2012): 429-440.

[17] Pennec, X., Fillard, P., Ayache, N. (2006). A Riemannian framework for tensor computing. International Journal of Computer Vision, 66(1), 41-66. 
[18] Provenzi, E. (2016). A differential geometry model for the perceived colors space. International Journal of Geometric Methods in Modern Physics, 13(08), 1630008.

[19] Resnikoff, H. L. (1974). Differential geometry and color perception. Journal of Mathematical Biology, 1(2), 97-131.

[20] Schrödinger, E. (1920). Grundlinien einer Theorie der Farbenmetrik im Tagessehen. Annalen der Physik, 368(22), 481-520.

[21] Silberstein, L. (1943). Investigations on the intrinsic properties of the color domain. II. JOSA, 33(1), 1-10.

[22] Wyszecki, G. and Stiles, W. S. (1982). Color science (ch. 8). New York: Wiley. 